\title{
One dimensional electromagnetic relativistic PIC-hydrodynamic hybrid simulation code H-VLPL (Hybrid Virtual Laser Plasma Lab)
}

\author{
Jalo Liljo ${ }^{1},{ }^{*}$ Anupam Karmakar ${ }^{\dagger}{ }^{\dagger}$ A. Pukhov ${ }^{2}$, and M. Hochbruck ${ }^{1}$ \\ ${ }^{1}$ Mathematisches Institut, Heinrich-Heine Universität, 40225, Düsseldorf, Germany \\ ${ }^{2}$ Institut für Theoretische Physik I, Heinrich-Heine Universität, 40225, Düsseldorf, Germany
}

In this paper we present a new one dimensional full electromagnetic relativistic hybrid plasma model. The full kinetic particle-in cell (PIC) and hydrodynamic model have been combined in the single hybrid plasma code H-VLPL (hybrid virtual laser plasma laboratory). The semi-implicit algorithm allows to simulate plasmas of arbitrary densities via automatic reduction of the highest plasma frequencies down to the numerically stable range. At the same time, the model keeps the correct spatial scales like the plasma skin depth. We discuss the numerically efficient implementation of this model. Further, we carefully test the hybrid model validity by applying it to a series of physical examples. The new mathematical method allows to overcome the typical time step restrictions of explicit PIC codes.

PACS numbers: 52.65.-y, 52.65.Rr; 52.65.Kj, 52.65.Ww, 52.65.Kd

Keywords: Plasma simulation; Object-oriented programming; Particle-in-Cell; hydrodynamic description; Hybrid simulation; Laser plasma interactions

\section{INTRODUCTION}

Computer simulations are key tools in the study of laser-plasma interactions [1, 2]. Large full 3D parallel electromagnetic simulation codes like VLPL [3], OSIRIS [4], VORPAL [5], OOPIC [6], and others contributed remarkably in our understanding of the complex laser-plasma physics. Adding new physical processes in the codes, as well as doing large scale high density plasma simulations are becoming more and more computationally expensive. As a result new algorithms and simulation techniques are being developed to cope with challenges of the laser-plasma physics.

One of the reasons why the classical explicit particle-in-cell (PIC) methods are extremely computationally expensive is that they have to resolve the plasma frequency $\omega_{p}=\sqrt{4 \pi n_{e} e^{2} / m_{e}}$, which is the frequency of the plasma electrostatic oscillations. Therefore, they are limited to be applied to systems with low plasma densities only.

On the contrary, there is a demand to simulate high density plasmas, e.g., in the experiments where the laser pulse interacts with solid targets [7]. The solid state density plasmas densities vary over a range $100-1000 n_{c}$, where $n_{c}=m \omega^{2} / 4 \pi e^{2}$ is the critical plasma density. Here, $m$ is the electron mass, $-e$ is its charge, and $\omega$ is the laser frequency. Other important applications include the Fast Ignition (FI) physics in the Inertial Confinement Fusion (ICF) studies [8]. The FI plasma has a density of the 1000 times compressed solid hydrogen, i.e., of the order of $10^{5} n_{c}$. Hence, the applicability of the classical PIC codes in this density range is facing a big question. In this situation, one is forced to look for a more efficient numerical method to challenge those ultra-high densities. One of the possibilities is to include hydrodynamic description of the high density plasma in the fully kinetic PIC code.

In last couple of years PIC-hydrodynamic hybrid techniques have emerged as an efficient solution to large scale ultra high-density plasma simulations, e.g., FI physics, solid state density plasma interactions, high charge, high energy ion generations etc $[9,12,13]$. Most of these codes work in the Darwin approximation and thus exclude the electromagnetic wave propagation completely. They also exclude electrostatic waves keeping the collisional magnetohydrodynamics (MHD) only. Further, implicit electrostatic particle-fluid hybrid plasma code has been developed by Rambo and Denavit [10], which has been used to study interpenetration and ion separation in colliding plasmas [11]. There is also the implicit electromagnetic PIC code LSP [14]. This code uses an implicit global scheme which leads to no such restrictions over time-step. The LSP code also employs a field solver based on an unconditionally Courant-stable algorithm[15] for electromagnetic calculations.

The newly developed code Hybrid Virtual Laser Plasma Laboratory (H-VLPL) is a hybrid code which unites a hydrodynamic model for overdense plasmas and the full kinetic description of hot low-density electrons and ions. The schematics of the code is shown in Fig. 1.

\footnotetext{
*Electronic address: liljo@am.uni-duesseldorf.de

$\dagger$ Electronic address: anupam@tp1.uni-duesseldorf.de
} 


\section{Hybrid Code for Relativistic Laser-Plasma}

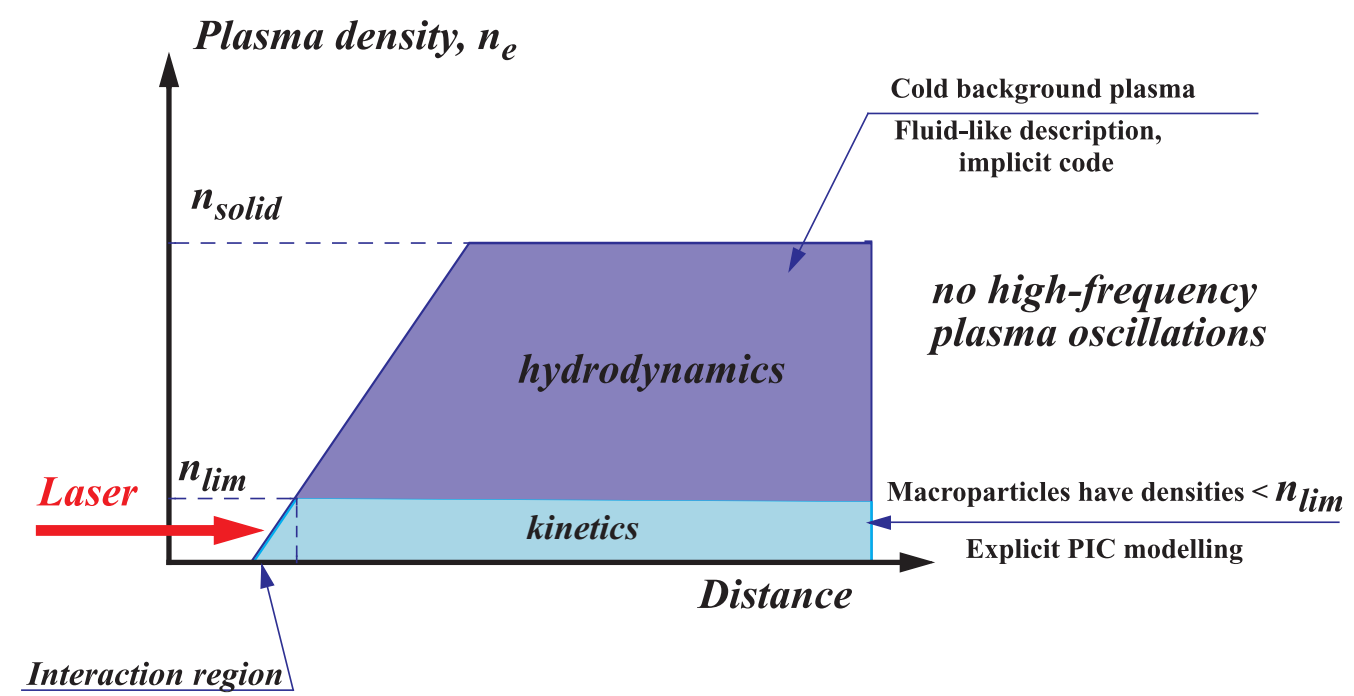

FIG. 1: (color) Schematics of the code H-VLPL. Low-density plasma is described kinetically using the explicit electromagnetic PIC technique. The cold overdense background plasma is described hydrodynamically. The full electromagnetic field solver is applied to the complete domain of simulations, also in the region of overdense plasma.

New matrix algorithms are developed to eradicate the time-step restrictions due to high plasma frequencies. Since the density of hot electrons is typically rather low, hybrid codes are expected to be more efficient than direct PIC codes. This allows for simulations with physically relevant solid state densities. Although the code H-VLPL automatically reduces the highest numerical frequency to the stable range, the spatial description of the field distribution remains correct. Even when the grid step is much larger than the plasma skin depth, the algorithm gives the correct exponential decay of electromagnetic fields in overdense plasma layer (see Section V).

The paper is organized as follows. First, we describe the full hybrid method, Section II. Then, we write down the implicit numerical scheme and provide the computationally efficient matrix algorithm to solve the implicit set of equations, Section III. In Section IV, we study the numerical dispersion relation for the new scheme and check its stability. Finally, we test the new code H-VLPL extensively on a number of well-known physical examples, Section $\mathrm{V}$.

\section{HYBRID MODEL}

We begin with writing down the master equations on the fields and particle momenta:

$$
\begin{aligned}
\frac{\partial \vec{E}}{\partial t} & =c \nabla \times \vec{B}-4 \pi \sum_{\ell} \vec{J}_{\ell}, \quad \ell=e, i, h \\
\frac{\partial \vec{B}}{\partial t} & =-c \nabla \times \vec{E} \\
\frac{d \vec{p}_{h}}{d t} & =q_{e} \vec{E}-\nu m \vec{v}_{h} \\
\frac{d \vec{p}_{\ell}}{d t} & =q_{\ell}\left(\vec{E}+\frac{\vec{v}_{\ell}}{c} \times \vec{B}\right), \quad \ell=e, i
\end{aligned}
$$

where

$$
\vec{J}_{\ell}=q_{\ell} n_{\ell} \vec{v}_{\ell}, \quad \vec{p}_{\ell}=m_{\ell} \gamma_{\ell} \vec{v}_{\ell}, \quad \gamma_{\ell}=\sqrt{1+\frac{p_{\ell}^{2}}{\left(m_{\ell} c\right)^{2}}}, \quad \nu=\eta n_{h}
$$


The index $\ell=e, i, h$ stays for electrons, ions, and hybrid particles respectively. $\vec{E}$ and $\vec{B}$ denote the electric and magnetic field vectors, $\vec{J}$ denotes the current density, $\vec{p}$ is the momentum and $n$ is the number density of particles. The parameter $\nu$ stays for collision frequency that defines the cold plasma conductivity.

Eqs. (1a)-(1b) show that we use the unabridged Maxwell equations and thus include the full physics of electromagnetic waves. Eq. (1d) corresponds to the fully kinetic algorithm for the low density electrons and ions.

We have to explain the meaning of Eq. (1c). The "hybrid particles" are assumed to be compound quasineutral objects, i.e., the negative charge of electrons within the hybrid particles is fully compensated by the positive charge of ions. The electrostatic force of "hybrid ions" is so strong that "hybrid electrons" cannot be separated and the hybrid particle moves as a whole. At the same time, electrons within the hybrid particle are allowed to have non-zero momenta $\vec{p}_{h}$ and to generate currents $\overrightarrow{j_{h}}=-e n_{h} \overrightarrow{v_{h}}$. This kind of plasma description corresponds to the single fluid MHD model [16]. Because the "hybrid electrons" are assumed to move slowly, $v_{h} \ll c$, and we are interested in their response to fast electric fields only, hence, we neglect the $\vec{v} \times \vec{B} / c$ term in the Lorentz force (1c).

For the kinetic part we use the standard Cloud-In-Cell (CIC) interpolation scheme. On the other hand, the hybrid particles are presently point like and are treated with Nearest Grid Point (NGP) interpolations. One has to mention here that, the kinetic part of the code exploits the energy conservative scheme, which has been benchmarked later in Section V.

\section{THE NUMERICAL ALGORITHM OF H-VLPL}

For simplicity, we rewrite the equations in dimensionless variables, $\widetilde{t}=\omega_{0} t$ and $\widetilde{x}=k_{0} x$, where $\omega_{0}$ denotes the laser frequency and $k_{0}=\omega_{0} / c$. The new variables are then

$$
\widetilde{E}=\frac{e E}{m_{e} c \omega_{0}}, \quad \widetilde{B}=\frac{e B}{m_{e} c \omega_{0}}, \quad \widetilde{p}_{\ell}=\frac{p_{\ell}}{m_{\ell} c}, \quad \widetilde{J}_{\ell}=\frac{J_{\ell}}{j_{c}}, \quad j_{c}=e n_{c} c, \quad \widetilde{\rho}=\frac{n}{n_{c}}, \quad \widetilde{q}_{\ell}=\frac{q_{\ell}}{q_{e}} .
$$

In Sections III and IV we choose the $\rho$ notation instead of the usual $n$ for the number densities of particles to avoid a possible confusion with the time step number $n$.

In the following, we omit the tildes. Eq. (1) then reads

$$
\begin{aligned}
\frac{\partial \vec{E}}{\partial t} & =\nabla \times \vec{B}-\sum_{\ell} \vec{J}_{\ell}, \quad \ell=e, i, h \\
\frac{\partial \vec{B}}{\partial t} & =-\nabla \times \vec{E} \\
\frac{d \vec{p}_{h}}{d t} & =\vec{E}-\nu \vec{v}_{h} \\
\frac{d \vec{p}_{\ell}}{d t} & =q_{\ell}\left(\vec{E}+\vec{v}_{\ell} \times \vec{B}\right), \quad \ell=e, i .
\end{aligned}
$$

Suitable boundary conditions for the simulation of a laser-plasma interaction are inhomogeneous, time dependent Dirichlet boundary conditions for the incoming laser(s).

We consider the problem in one space dimension, i.e. all vectors are of the form

$$
\vec{V}=\left[V_{x}(t, x), V_{y}(t, x), V_{z}(t, x)\right]^{T}, \quad x \in[0, L]
$$

We thus have $B_{x}(t, x)=0$.

Following [1] we define

$$
F_{y}^{ \pm}=\frac{1}{2}\left(E_{y} \pm B_{z}\right), \quad F_{z}^{ \pm}=\frac{1}{2}\left(E_{z} \pm B_{y}\right)
$$

Then $(2 \mathrm{a})-(2 \mathrm{c})$ are equivalent to 


$$
\begin{aligned}
\frac{\partial F_{y}^{ \pm}}{\partial t} & =\mp \frac{\partial F_{y}^{ \pm}}{\partial x}-\frac{1}{2} \rho_{h} v_{h, y}-\frac{1}{2} J_{e, y} \\
\frac{\partial F_{z}^{ \pm}}{\partial t} & = \pm \frac{\partial F_{z}^{ \pm}}{\partial x}-\frac{1}{2} \rho_{h} v_{h, z}-\frac{1}{2} J_{e, z} \\
\frac{d p_{h, y}}{d t} & =F_{y}^{+}+F_{y}^{-}-\eta \rho_{h} v_{h, y} \\
\frac{d p_{h, z}}{d t} & =F_{z}^{+}+F_{z}^{-}-\eta \rho_{h} v_{h, z} .
\end{aligned}
$$

The boundary conditions are given by $F_{y}^{+}(0)=F_{z}^{-}(0)=g(t)$ and $F_{y}^{-}(L)=F_{z}^{+}(L)=0$.

For the numerical discretization we use an equidistant staggered grid in space and time with spatial step size $h=L / m$ for some positive integer $m$ and temporal step size $\tau>0$. The fields at grid point $j$ and time $t_{n}=n \tau$ are denoted by $F_{j}^{n}, j=0, \ldots, m, n \geq 0$. The field equations are integrated along the vacuum characteristics ( $x \mp t=$ constant) which implies $h=\tau$. We discretize the fields $E$ and $B$ and the momenta $p_{h}, p_{e}$. The current $\vec{J}_{h}$ and the velocity are computed via $\vec{J}_{h}=\rho_{h} \vec{p}_{h} / \gamma_{h}=\rho_{h} \vec{v}_{h}$. Fig. 2 shows the staggered grid and the location of the variables.

We suggest the following implicit finite difference scheme for solving (4):

$$
\begin{aligned}
& \frac{\left(E_{x}\right)_{j}^{n+1}-\left(E_{x}\right)_{j}^{n}}{\tau}=-\left(\rho_{h} v_{h, x}\right)_{j}^{n+\frac{1}{2}}-\left(J_{e, x}\right)_{j}^{n+\frac{1}{2}} \\
& \frac{\left(p_{h, x}\right)_{j}^{n+1}-\left(p_{h, x}\right)_{j}^{n}}{\tau}=\frac{\left(E_{x}\right)_{j}^{n+1}+\left(E_{x}\right)_{j}^{n}}{2}-\eta\left(\rho_{h} v_{h, x}\right)_{j}^{n+1} \\
& \frac{\left(F_{y}^{+}\right)_{j+1}^{n+1}-\left(F_{y}^{+}\right)_{j+1}^{n}}{\tau}=-\frac{\left(F_{y}^{+}\right)_{j+1}^{n}-\left(F_{y}^{+}\right)_{j}^{n}}{h}-\frac{1}{2}\left(\rho_{h} v_{h, y}\right)_{j+\frac{1}{2}}^{n+\frac{1}{2}}-\frac{1}{2}\left(J_{e, y}\right)_{j+\frac{1}{2}}^{n+\frac{1}{2}} \\
& \frac{\left(F_{y}^{-}\right)_{j}^{n+1}-\left(F_{y}^{-}\right)_{j}^{n}}{\tau}=\frac{\left(F_{y}^{-}\right)_{j+1}^{n}-\left(F_{y}^{-}\right)_{j}^{n}}{h}-\frac{1}{2}\left(\rho_{h} v_{h, y}\right)_{j+\frac{1}{2}}^{n+\frac{1}{2}}-\frac{1}{2}\left(J_{e, y}\right)_{j+\frac{1}{2}}^{n+\frac{1}{2}} \\
& \frac{\left(p_{h, y}\right)_{j}^{n+1}-\left(p_{h, y}\right)_{j}^{n}}{\tau}=\frac{\left(F_{y}^{+}\right)_{j}^{n+1}+\left(F_{y}^{+}\right)_{j}^{n}+\left(F_{y}^{-}\right)_{j}^{n+1}+\left(F_{y}^{-}\right)_{j}^{n}}{2} \\
& \frac{\left(F_{z}^{+}\right)_{j}^{n+1}-\left(F_{z}^{+}\right)_{j}^{n}}{\tau}=\frac{\left(F_{z}^{+}\right)_{j+1}^{n}-\left(F_{z}^{+}\right)_{j}^{n}}{h}-\frac{1}{2}\left(\rho_{h} v_{h, z}\right)_{j+\frac{1}{2}}^{n+\frac{1}{2}}-\frac{1}{2}\left(J_{e, z}\right)_{j+\frac{1}{2}}^{n+\frac{1}{2}} \\
& \frac{\left(F_{z}^{-}\right)_{j+1}^{n+1}-\left(F_{z}^{-}\right)_{j+1}^{n}}{\tau}=-\frac{\left(F_{z}^{-}\right)_{j+1}^{n}-\left(F_{z}^{-}\right)_{j}^{n}}{h}-\frac{1}{2}\left(\rho_{h} v_{h, z}\right)_{j+\frac{1}{2}}^{n+\frac{1}{2}}-\frac{1}{2}\left(J_{e, z}\right)_{j+\frac{1}{2}}^{n+\frac{1}{2}} \\
& \frac{\left(p_{h, z}\right)_{j}^{n+1}-\left(p_{h, z}\right)_{j}^{n}}{\tau}= \frac{\left(F_{z}^{+}\right)_{j}^{n+1}+\left(F_{z}^{+}\right)_{j}^{n}+\left(F_{z}^{-}\right)_{j}^{n+1}+\left(F_{z}^{-}\right)_{j}^{n}}{2} \\
&-\eta\left(\rho_{h} v_{h, z}\right)_{j}^{n+1},
\end{aligned}
$$

Within this scheme, we approximate

$$
\begin{aligned}
\left(\rho_{h} v_{h, x}\right)_{j}^{n+\frac{1}{2}}= & \frac{\left(\zeta_{h}\right)_{j}^{n}}{2}\left(\left(p_{h, x}\right)_{j}^{n+1}+\left(p_{h, x}\right)_{j}^{n}\right) \\
\left(\rho_{h} v_{h, s}\right)_{j+\frac{1}{2}}^{n+\frac{1}{2}}= & \left.\frac{\left(\zeta_{h}\right)_{j}^{n}}{4}\left(\left(p_{h, s}\right)_{j}^{n+1}+\left(p_{h, s}\right)_{j}^{n}\right)\right) \\
& +\frac{\left(\zeta_{h}\right)_{j+1}^{n}}{4}\left(\left(p_{h, s}\right)_{j+1}^{n+1}+\left(p_{h, s}\right)_{j+1}^{n}\right), \quad s=y, z \\
\left(\rho_{h} v_{h, s}\right)_{j}^{n+1}= & \left(\zeta_{h}\right)_{j}^{n}\left(p_{h, s}\right)_{j}^{n+1}, \quad s=x, y, z,
\end{aligned}
$$

where $\zeta_{h}=\rho_{h} / \gamma_{h}$. This leads to the following scheme for the $x$-component 


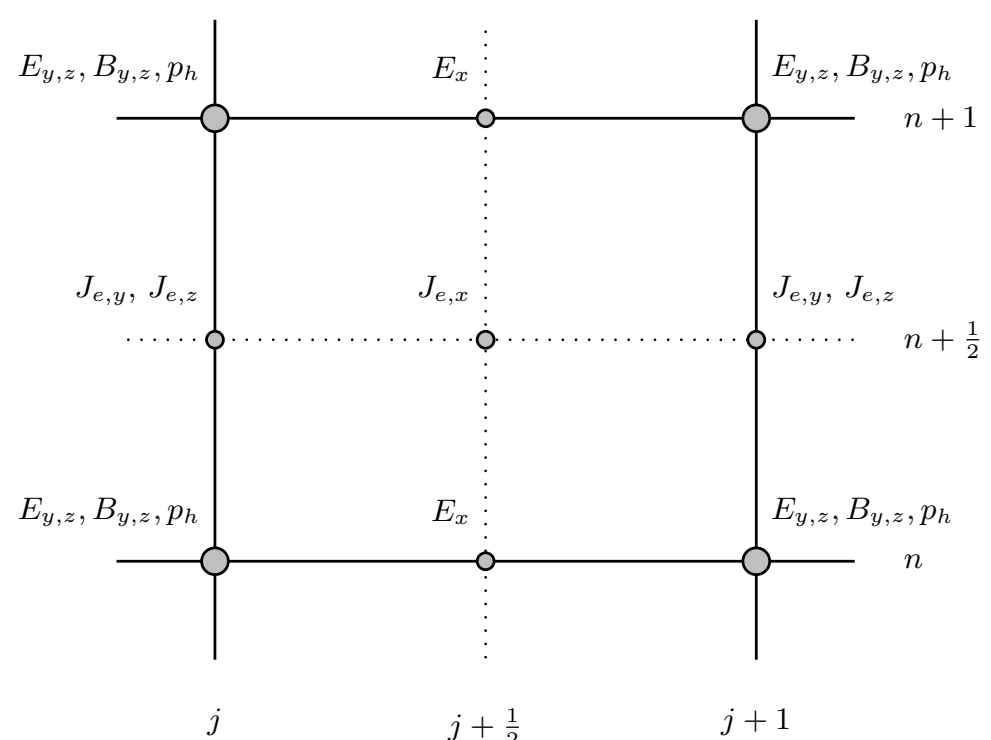

FIG. 2: Staggered grid for the finite difference scheme (5).

$$
\begin{aligned}
\left(E_{x}\right)_{j}^{n+1}= & \frac{1}{\left(K^{+}\right)_{j}^{n}}\left(\left(K^{-}\right)_{j}^{n}\left(E_{x}\right)_{j}^{n}-\frac{\tau\left(\zeta_{h}\right)_{j}^{n}\left(p_{h, x}\right)_{j}^{n}}{2}\left(1+\frac{1}{1+\tau \eta\left(\zeta_{h}\right)_{j}^{n}}\right)\right. \\
& \left.-\tau\left(J_{e, x}\right)_{j}^{n+\frac{1}{2}}\right) \\
\left(p_{h, x}\right)_{j}^{n+1}= & \frac{\left(p_{h, x}\right)_{j}^{n}+\frac{\tau}{2}\left(\left(E_{x}\right)_{j}^{n+1}+\left(E_{x}\right)_{j}^{n}\right)}{1+\tau \eta\left(\zeta_{h}\right)_{j}^{n}},
\end{aligned}
$$

where

$$
K^{ \pm}=1 \pm \frac{\tau^{2} \zeta_{h}}{4\left(1+\tau \eta \zeta_{h}\right)} .
$$

However, for the $y$-component we obtain the implicit scheme

$$
\begin{aligned}
\left(F_{y}^{+}\right)_{j+1}^{n+1}+ & \frac{\tau\left(\zeta_{h}\right)_{j}^{n}}{8}\left(p_{h, y}\right)_{j}^{n+1}+\frac{\tau\left(\zeta_{h}\right)_{j+1}^{n}}{8}\left(p_{h, y}\right)_{j+1}^{n+1} \\
= & \left(F_{y}^{+}\right)_{j}^{n}-\frac{\tau\left(\zeta_{h}\right)_{j}^{n}}{8}\left(p_{h, y}\right)_{j}^{n}-\frac{\tau\left(\zeta_{h}\right)_{j+1}^{n}}{8}\left(p_{h, y}\right)_{j+1}^{n}-\frac{\tau}{2}\left(J_{e, y}\right)_{j+\frac{1}{2}}^{n+\frac{1}{2}} \\
\left(F_{y}^{-}\right)_{j}^{n+1}+ & \frac{\tau\left(\zeta_{h}\right)_{j}^{n}}{8}\left(p_{h, y}\right)_{j}^{n+1}+\frac{\tau\left(\zeta_{h}\right)_{j+1}^{n}}{8}\left(p_{h, y}\right)_{j+1}^{n+1} \\
= & \left(F_{y}^{-}\right)_{j+1}^{n}-\frac{\tau\left(\zeta_{h}\right)_{j}^{n}}{8}\left(p_{h, y}\right)_{j}^{n}-\frac{\tau\left(\zeta_{h}\right)_{j+1}^{n}}{8}\left(p_{h, y}\right)_{j+1}^{n}-\frac{\tau}{2}\left(J_{e, y}\right)_{j+\frac{1}{2}}^{n+\frac{1}{2}} \\
(1+\tau \eta \quad & \left.\left(\zeta_{h}\right)_{j}^{n}\right)\left(p_{h, y}\right)_{j}^{n+1}-\frac{\tau}{2}\left(F_{y}^{+}\right)_{j}^{n+1}-\frac{\tau}{2}\left(F_{y}^{-}\right)_{j}^{n+1} \\
= & \left(p_{h, y}\right)_{j}^{n}+\frac{\tau}{2}\left(F_{y}^{+}\right)_{j}^{n}-\frac{\tau}{2}\left(F_{y}^{-}\right)_{j}^{n} .
\end{aligned}
$$

Next we consider the efficient solution of the linear system for the $y$-component. For $Y=$ $\left[\left(F_{y}^{+}\right)_{1}, \ldots,\left(F_{y}^{+}\right)_{m},\left(F_{y}^{-}\right)_{0}, \ldots,\left(F_{y}^{-}\right)_{m-1},\left(p_{h, y}\right)_{0}, \ldots,\left(p_{h, y}\right)_{m}\right]^{T}$ we obtain

$$
A Y^{n+1}=B Y^{n}+f(t),
$$

where $f$ contains the boundary conditions and the electron currents (from the PIC code), and 


$$
A=\left[\begin{array}{c|c}
I_{2 m} & D \\
\hline C & G_{1}
\end{array}\right] \quad \text { and } \quad B=\left[\begin{array}{cc|c}
S_{m}^{T} & 0 & -D \\
0 & S_{m} & \\
\hline-C & G_{2}
\end{array}\right]
$$

Here $I_{k}$ denotes the $k \times k$ identity matrix and $G_{1,2}=\operatorname{diag}\left(1 \pm \tau \eta\left(\zeta_{h}\right)_{j}^{n}\right) \in \mathbb{R}^{(m+1) \times(m+1)}$. $S_{k}$ represents the $k \times k$ shift matrix with ones on the first upper diagonal and zeros elsewhere. $C \in \mathbb{R}^{(m+1) \times 2 m}$ and $D \in \mathbb{R}^{2 m \times(m+1)}$ are given by

$$
C=-\frac{\tau}{2}\left[\begin{array}{c|c}
0 & I_{m} \\
I_{m} & 0
\end{array}\right], \quad D=\frac{\tau}{8}\left[\begin{array}{l}
\operatorname{bidiag}\left(\left(\zeta_{h}\right)_{j}^{n},\left(\zeta_{h}\right)_{j+1}^{n}\right)_{j=0}^{m-1}, \\
\operatorname{bidiag}\left(\left(\zeta_{h}\right)_{j}^{n},\left(\zeta_{h}\right)_{j+1}^{n}\right)_{j=0}^{m-1}
\end{array}\right]
$$

where bidiag $\left(a_{j}, b_{j}\right)_{j=1, m}$ denotes an $m \times(m+1)$ bidiagonal matrix with $a_{j}$ as the $j$ th diagonal entry and $b_{j}$ as the $j$ th upper diagonal entry.

This large linear system can be solved efficiently by using block Gaussian elimination

$$
A=\left[\begin{array}{c|c}
I_{2 m} & D \\
\hline C & G_{1}
\end{array}\right]=\left[\begin{array}{c|c}
I_{2 m} & 0 \\
\hline C & I_{m+1}
\end{array}\right]\left[\begin{array}{c|c}
I_{2 m} & D \\
\hline 0 & T
\end{array}\right],
$$

where $T=G_{1}-C D$ is the tridiagonal matrix containing the Schur complement [17] of $A$. Thus the solution of (9) can be reduced to solving a linear system with the tridiagonal matrix $T$ of size $m+1$.

Finally, the scheme for the $z$-component is given by

$$
\begin{aligned}
\left(F_{z}^{+}\right)_{j}^{n+1} & +\frac{\tau\left(\zeta_{h}\right)_{j}^{n}}{8}\left(p_{h, z}\right)_{j}^{n+1}+\frac{\tau\left(\zeta_{h}\right)_{j+1}^{n}}{8}\left(p_{h, z}\right)_{j+1}^{n+1} \\
& =\left(F_{z}^{+}\right)_{j+1}^{n}-\frac{\tau\left(\zeta_{h}\right)_{j}^{n}}{8}\left(p_{h, z}\right)_{j}^{n}-\frac{\tau\left(\zeta_{h}\right)_{j+1}^{n}}{8}\left(p_{h, z}\right)_{j+1}^{n}-\frac{\tau}{2}\left(J_{e, z}\right)_{j+\frac{1}{2}}^{n+\frac{1}{2}} \\
\left(F_{z}^{-}\right)_{j+1}^{n+1}+ & \frac{\tau\left(\zeta_{h}\right)_{j}^{n}}{8}\left(p_{h, z}\right)_{j}^{n+1}+\frac{\tau\left(\zeta_{h}\right)_{j+1}^{n}}{8}\left(p_{h, z}\right)_{j+1}^{n+1} \\
= & \left(F_{z}^{-}\right)_{j}^{n}-\frac{\tau\left(\zeta_{h}\right)_{j}^{n}}{8}\left(p_{h, z}\right)_{j}^{n}-\frac{\tau\left(\zeta_{h}\right)_{j+1}^{n}}{8}\left(p_{h, z}\right)_{j+1}^{n}-\frac{\tau}{2}\left(J_{e, z}\right)_{j+\frac{1}{2}}^{n+\frac{1}{2}} \\
(1+\tau \eta= & \left.\left(\zeta_{h}\right)_{j}^{n}\right)\left(p_{h, z}\right)_{j}^{n+1}-\frac{\tau}{2}\left(F_{z}^{+}\right)_{j}^{n+1}-\frac{\tau}{2}\left(F_{z}^{-}\right)_{j}^{n+1} \\
= & \left(p_{h, z}\right)_{j}^{n}+\frac{\tau}{2}\left(F_{z}^{+}\right)_{j}^{n}-\frac{\tau}{2}\left(F_{z}^{-}\right)_{j}^{n} .
\end{aligned}
$$

The resulting linear system is solved analogously to the one for the $y$-component.

\section{NUMERICAL DISPERSION AND STABILITY}

In this section we will derive the dispersion relations for the hybrid scheme (5) applied to the dimensionless equations (2). In dimensionless variables the plasma frequency is given by

$$
\widetilde{\omega}_{\ell}=\omega_{\ell} / \omega_{0}=\sqrt{\widetilde{\rho}_{\ell}}, \quad \ell=e, h .
$$

We again omit the tildes, set $\gamma_{\ell}=1$ and $\rho_{\ell}=$ const. The explicit PIC scheme is stable for step sizes

$$
\tau \leq \frac{2}{\omega_{e}}
$$

Therefore it is prohibitive to use this scheme for high densities. 


\section{A. Dispersion relation for the $x$-component}

Due to $B_{x}(t, x)=0$ we have

$$
\begin{aligned}
\frac{\partial \vec{E}}{\partial t} & =-\rho_{h} \vec{p}_{h}-\rho_{e} \vec{p}_{e} \\
\frac{\partial \vec{p}_{h}}{\partial t} & =\vec{E}-\nu \vec{v}_{h} \\
\frac{\partial \vec{p}_{e}}{\partial t} & =\vec{E}
\end{aligned}
$$

to obtain the dispersion relation for the $x$-component.

The finite difference scheme of (13) is given by

$$
\begin{aligned}
\frac{\left(E_{x}\right)_{j}^{n+1}-\left(E_{x}\right)_{j}^{n}}{\tau} & =-\rho_{h} \frac{\left(p_{h, x}\right)_{j}^{n+1}+\left(p_{h, x}\right)_{j}^{n}}{2}-\rho_{e}\left(p_{e, x}\right)_{j}^{n+\frac{1}{2}} \\
\frac{\left(p_{h, x}\right)_{j}^{n+1}-\left(p_{h, x}\right)_{j}^{n}}{\tau} & =\frac{\left(E_{x}\right)_{j}^{n+1}+\left(E_{x}\right)_{j}^{n}}{2}-\eta \rho_{h}\left(p_{h, x}\right)_{j}^{n+1} \\
\frac{\left(p_{e, x}\right)_{j}^{n+\frac{1}{2}}-\left(p_{e, x}\right)_{j}^{n-\frac{1}{2}}}{\tau} & =\left(E_{x}\right)_{j}^{n} .
\end{aligned}
$$

Substituting plane waves

$$
\begin{aligned}
\left(E_{x}\right)_{j}^{n} & =E_{0} e^{i(\omega n \tau-k j h)}, \\
\left(p_{h, x}\right)_{j}^{n} & =\left(p_{h}\right)_{0} e^{i(\omega n \tau-k j h)}, \\
\left(p_{e, x}\right)_{j}^{n} & =\left(p_{e}\right)_{0} e^{i(\omega n \tau-k j h)}
\end{aligned}
$$

into (14) and using (11) leads to

$$
\begin{aligned}
i E_{0} \sin \frac{\omega \tau}{2} & =-\frac{\tau \omega_{h}^{2}}{2}\left(p_{h}\right)_{0} \cos \frac{\omega \tau}{2}-\frac{\tau \omega_{e}^{2}}{2}\left(p_{e}\right)_{0}, \\
i\left(p_{h}\right)_{0} \sin \frac{\omega \tau}{2} & =-\frac{\tau}{2} E_{0} \cos \frac{\omega \tau}{2}-\frac{\tau \eta \omega_{h}^{2}}{2}\left(p_{h}\right)_{0} e^{i \frac{\omega \tau}{2}}, \\
i\left(p_{e}\right)_{0} \sin \frac{\omega \tau}{2} & =\tau E_{0} .
\end{aligned}
$$

Solving $(16 \mathrm{~b})$ for $\left(p_{h}\right)_{0},(16 \mathrm{c})$ for $\left(p_{e}\right)_{0}$ and inserting into (16a) yields

$$
\sin ^{2} \frac{\omega \tau}{2}=\frac{\tau^{2} \omega_{h}^{2}}{4} \frac{\cos ^{2} \frac{\omega \tau}{2} \sin \frac{\omega \tau}{2}}{\sin \frac{\omega \tau}{2}-i \frac{\tau \eta \omega_{h}^{2}}{2} e^{i \frac{\omega \tau}{2}}}+\frac{\tau^{2} \omega_{e}^{2}}{4}
$$

and for $\eta=0$ we obtain

$$
\omega=\frac{2}{\tau} \arccos \sqrt{\frac{1-\left(\frac{\tau \omega_{e}}{2}\right)^{2}}{1+\left(\frac{\tau \omega_{h}}{2}\right)^{2}}} .
$$

The numerical dispersion relation (18) shows that the scheme is unconditionally stable for $\tau \leq 2 / \omega_{e}$, i.e. independent of $\omega_{h}$. This step size restriction is due to the explicit PIC code. Note that in our hybrid model we have $\omega_{e} \ll \omega_{h}$, so this restriction is not severe.

For $\eta \neq 0$, the stability analysis is more involved since we have complex coefficients in the relation (18). In general, this leads to complex valued solutions $\omega$. Therefore, we verified numerically, that the scheme is stable for $\tau \in\left(0,2 / \omega_{e}\right)$ and $\eta \in[0,1]$. 


\section{B. Dispersion relation for the $y$ - and the $z$-component}

For the $y$-component we have

$$
\begin{aligned}
\frac{\partial F_{y}^{+}}{\partial t} & =-\frac{\partial}{\partial x} F_{y}^{+}-\frac{1}{2} \rho_{h} p_{h, y}-\frac{1}{2} J_{e, y} \\
\frac{\partial F_{y}^{-}}{\partial t} & =\frac{\partial}{\partial x} F_{y}^{-}-\frac{1}{2} \rho_{h} p_{h, y}-\frac{1}{2} J_{e, y} \\
\frac{\partial p_{h, y}}{\partial t} & =F_{y}^{+}+F_{y}^{-}-\eta \rho_{h} p_{h, y} \\
\frac{\partial p_{e, y}}{\partial t} & =E_{y}
\end{aligned}
$$

The finite difference scheme then reads

$$
\begin{aligned}
\left(F_{y}^{+}\right)_{j+1}^{n+1}-\left(F_{y}^{+}\right)_{j+1}^{n}= & -\left(F_{y}^{+}\right)_{j+1}^{n}+\left(F_{y}^{+}\right)_{j}^{n}-\frac{\tau}{2} \rho_{h}\left(p_{h, y}\right)_{j+\frac{1}{2}}^{n+\frac{1}{2}}-\frac{\tau}{2}\left(J_{e, y}\right)_{j+\frac{1}{2}}^{n+\frac{1}{2}} \\
\left(F_{y}^{-}\right)_{j}^{n+1}-\left(F_{y}^{-}\right)_{j}^{n}= & \left(F_{y}^{-}\right)_{j+1}^{n}-\left(F_{y}^{-}\right)_{j}^{n}-\frac{\tau}{2} \rho_{h}\left(p_{h, y}\right)_{j+\frac{1}{2}}^{n+\frac{1}{2}}-\frac{\tau}{2}\left(J_{e, y}\right)_{j+\frac{1}{2}}^{n+\frac{1}{2}} \\
\left(p_{h, y}\right)_{j}^{n+1}-\left(p_{h, y}\right)_{j}^{n}= & \frac{\tau}{2}\left(\left(F_{y}^{+}\right)_{j}^{n+1}+\left(F_{y}^{+}\right)_{j}^{n}+\left(F_{y}^{-}\right)_{j}^{n+1}+\left(F_{y}^{-}\right)_{j}^{n}\right) \\
& -\tau \eta \rho_{h}\left(p_{h, y}\right)_{j}^{n+1} \\
\left(p_{e, y}\right)_{j}^{n+\frac{1}{2}}-\left(p_{e, y}\right)_{j}^{n-\frac{1}{2}}= & \tau\left(E_{y}\right)_{j}^{n} .
\end{aligned}
$$

For the dispersion relation, we rewrite (20) in terms of the original fields $E$ and $B$ :

$$
\begin{aligned}
&\left(E_{y}\right)_{j+1}^{n+1}-\left(E_{y}\right)_{j}^{n}=-\left(B_{z}\right)_{j+1}^{n+1}+\left(B_{z}\right)_{j}^{n} \\
&-\tau \omega_{h}^{2} \frac{\left(p_{h, y}\right)_{j}^{n+1}+\left(p_{h, y}\right)_{j}^{n}+\left(p_{h, y}\right)_{j+1}^{n+1}+\left(p_{h, y}\right)_{j+1}^{n}}{4} \\
& \\
&-\tau \omega_{e}^{2} \frac{\left(p_{e, y}\right)_{j}^{n+\frac{1}{2}}+\left(p_{e, y}\right)_{j+1}^{n+\frac{1}{2}}}{2} \\
&\left(E_{y}\right)_{j}^{n+1}-\left(E_{y}\right)_{j+1}^{n}=\quad\left(B_{z}\right)_{j}^{n+1}-\left(B_{z}\right)_{j+1}^{n} \\
&-\tau \omega_{h}^{2} \frac{\left(p_{h, y}\right)_{j}^{n+1}+\left(p_{h, y}\right)_{j}^{n}+\left(p_{h, y}\right)_{j+1}^{n+1}+\left(p_{h, y}\right)_{j+1}^{n}}{4} \\
&-\tau \omega_{e}^{2} \frac{\left(p_{e, y}\right)_{j}^{n+\frac{1}{2}}+\left(p_{e, y}\right)_{j+1}^{n+\frac{1}{2}}}{2} \\
& \frac{\left(E_{y}\right)_{j}^{n+1}+\left(E_{y}\right)_{j}^{n}}{2}=\left(1+\tau \eta \omega_{h}^{2}\right)\left(p_{h, y}\right)_{j}^{n+1}-\left(p_{h, y}\right)_{j}^{n} \\
& \tau\left(E_{y}\right)_{j}^{n}=\quad\left(p_{e, y}\right)_{j}^{n+\frac{1}{2}}-\left(p_{e, y}\right)_{j}^{n-\frac{1}{2}} .
\end{aligned}
$$

Adding and subtracting (21a) and (21b) yields

$$
\begin{aligned}
\left(E_{y}\right)_{j+1}^{n+1}+\left(E_{y}\right)_{j}^{n+1} & -\left(E_{y}\right)_{j}^{n}-\left(E_{y}\right)_{j+1}^{n}=\left(B_{z}\right)_{j}^{n+1}+\left(B_{z}\right)_{j}^{n}-\left(B_{z}\right)_{j+1}^{n+1}-\left(B_{z}\right)_{j+1}^{n} \\
& -\tau \omega_{h}^{2} \frac{\left(p_{h, y}\right)_{j}^{n+1}+\left(p_{h, y}\right)_{j}^{n}+\left(p_{h, y}\right)_{j+1}^{n+1}+\left(p_{h, y}\right)_{j+1}^{n}}{2} \\
& -\tau \omega_{e}^{2}\left(\left(p_{e, y}\right)_{j}^{n+\frac{1}{2}}+\left(p_{e, y}\right)_{j+1}^{n+\frac{1}{2}}\right) \\
\left(E_{y}\right)_{j+1}^{n+1}+\left(E_{y}\right)_{j+1}^{n} & -\left(E_{y}\right)_{j}^{n+1}-\left(E_{y}\right)_{j}^{n}= \\
& -\left(B_{z}\right)_{j}^{n+1}+\left(B_{z}\right)_{j}^{n}-\left(B_{z}\right)_{j+1}^{n+1}+\left(B_{z}\right)_{j+1}^{n} .
\end{aligned}
$$

Analogously to (15) we substitute plane waves, which gives 


$$
\begin{aligned}
E_{0} \sin \frac{\omega \tau}{2} \cos \frac{k \tau}{2} & =-B_{0} \cos \frac{\omega \tau}{2} \sin \frac{k \tau}{2}+i\left(p_{h}\right)_{0} \frac{\tau \omega_{h}^{2}}{2} \cos \frac{\omega \tau}{2} \cos \frac{k \tau}{2}+i \frac{\tau \omega_{e}^{2}}{2} \\
E_{0} \sin \frac{k \tau}{2} \cos \frac{\omega \tau}{2} & =-B_{0} \sin \frac{\omega \tau}{2} \cos \frac{k \tau}{2} \\
\frac{\tau E_{0} \cos \frac{\omega \tau}{2}}{2} & =\left(p_{h}\right)_{0}\left(i \sin \frac{\omega \tau}{2}+\frac{\eta \tau \omega_{h}^{2} e^{i \frac{\omega \tau}{2}}}{2}\right) \\
\left(p_{e}\right)_{0} \sin \frac{\omega \tau}{2} & =\frac{\tau E_{0}}{2 i} .
\end{aligned}
$$

Solving $(23 \mathrm{~b})$ for $B_{0},(23 \mathrm{c})$ for $\left(p_{h}\right)_{0},(23 \mathrm{~d})$ for $\left(p_{e}\right)_{0}$ and inserting into (23a) yields

$$
\sin ^{2} \frac{\omega \tau}{2} \cos ^{2} \frac{k \tau}{2}=\cos ^{2} \frac{\omega \tau}{2} \sin ^{2} \frac{k \tau}{2}+\left(\frac{\tau \omega_{h}}{2}\right)^{2} \frac{\cos ^{2} \frac{\omega \tau}{2} \cos ^{2} \frac{k \tau}{2} \sin \frac{\omega \tau}{2}}{\sin \frac{\omega \tau}{2}-i \frac{\tau \eta \omega_{h}^{2}}{2} e^{i \frac{\omega \tau}{2}}}+\left(\frac{\tau \omega_{e}}{2}\right)^{2} \cos ^{2} \frac{k \tau}{2}
$$

from which we obtain

$$
\cos ^{2} \frac{\omega \tau}{2}\left(1+\frac{\tau^{2} \omega_{h}^{2}}{4} \frac{\cos ^{2} \frac{k \tau}{2} \sin \frac{\omega \tau}{2}}{\sin \frac{\omega \tau}{2}-i \frac{\tau \eta \omega_{h}^{2}}{2} e^{i \frac{\omega \tau}{2}}}\right)=\cos ^{2} \frac{k \tau}{2}\left(1-\frac{\tau^{2} \omega_{e}^{2}}{4}\right)
$$

For $\cos ^{2} \frac{k \tau}{2}=1$, this is the same dispersion relation as for the $x$-component. Again, we solved the relation numerically and verified stability for $\tau \in\left(0,2 / \omega_{e}\right)$, and $\cos ^{2} \frac{k \tau}{2} \in[0,1]$.

For $\eta=0$, the dispersion relation reads

$$
\cos ^{2} \frac{\omega \tau}{2}=\frac{\cos ^{2} \frac{k \tau}{2}\left(1-\left(\frac{\tau \omega_{e}}{2}\right)^{2}\right)}{1+\left(\frac{\tau \omega_{h}}{2}\right)^{2} \cos ^{2} \frac{k \tau}{2}}=: \xi^{2}
$$

and we obtain

$$
\omega=\frac{2}{\tau} \arccos \xi
$$

This shows that the scheme is unconditionally stable for $\tau \leq 2 / \omega_{e}$.

In Fig. 3 analytical plots for the real and imaginary parts of $k(\omega)$ are presented for $\eta=0, \omega_{e}=0, \omega_{h}=31.6, \tau=0.05$ and $\omega \in\left[0, \frac{\pi}{2 \tau}\right]$.

Analogously, one can obtain the same relation for the $z$-component.

\section{BENCHMARKING WITH PHYSICAL PROCESSES}

The numerical scheme described in the previous sections is implemented in the code H-VLPL. This section describes key benchmark tests to evaluate accuracy and applicability of the scheme. First, we check the reflection, transmission and refraction of a laser pulse at a hybrid plasma slab. Second, we verify the validity of the energy conservation in the present algorithm. Third, we check the well known process of Target Normal Sheath Acceleration (TNSA)[18, 19]. Fourth, we benchmark plasma skin fields at very high hybrid densities when the spatial grid cell size is much larger than the plasma skin length. Lastly, we check absorption of a circularly polarized Gaussian laser pulse over a long distance propagation in underdense hybrid plasmas. Wherever possible, we compare the numerical results with existing analytic solutions.

\section{A. Reflection and refraction of incident pulse}

The first and the simplest trial one can think of is the full reflection of the incident laser pulse from the surface of overdense plasma, as well as, transmission and reflection of the same when the plasma is underdense. In Fig. 4(a) a 

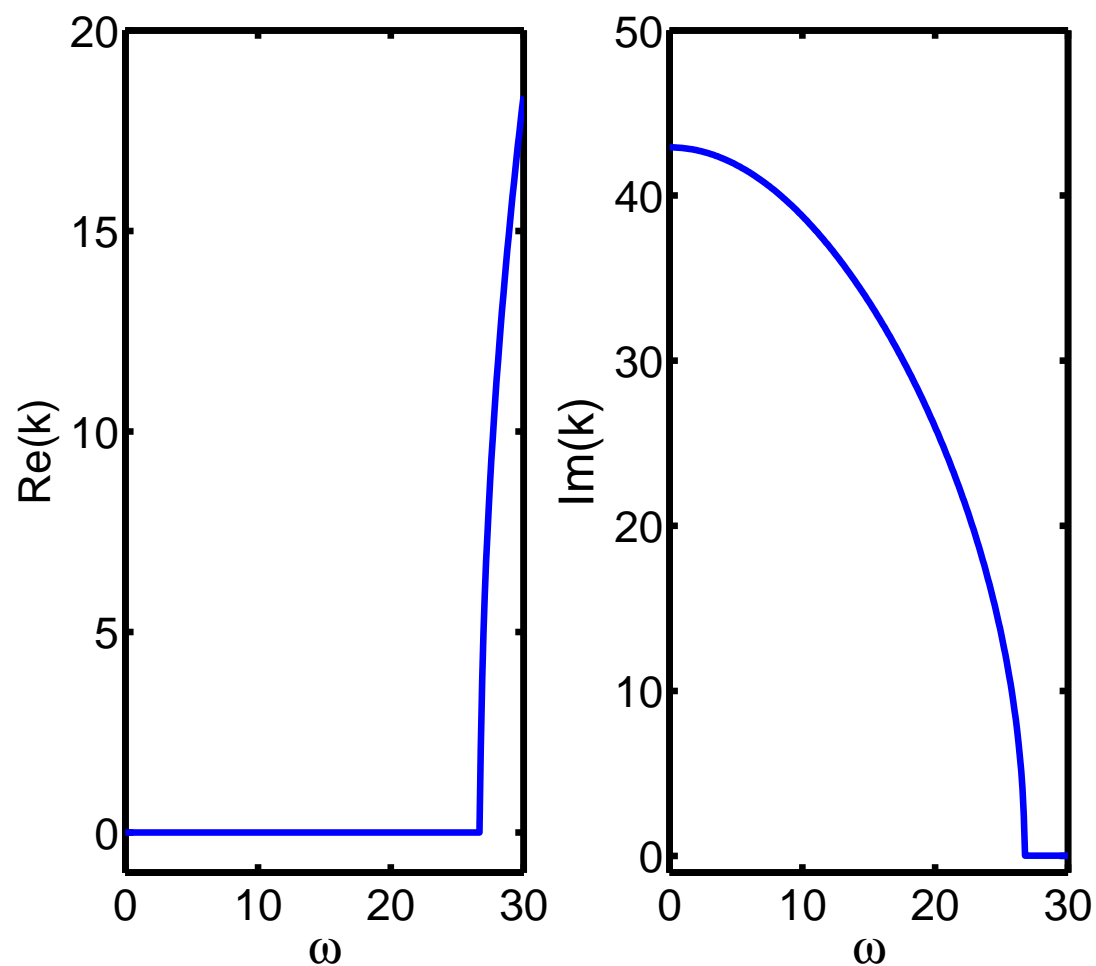

FIG. 3: (color) Plot of dispersion relation for real and imaginary part of $k(\omega)$ with $\eta=0, \omega_{e}=0, \omega_{h}=31.6, \tau=0.05$ and $\omega \in\left[0, \frac{\pi}{2 \tau}\right]$. The simulation for the same case with $\omega_{h}=31.6$ will be discussed in Section V and corresponding Fig. 10.

$30 \mathrm{fs}$ Gaussian laser pulse propagates in the positive $x$ direction towards an underdense $\left(n=0.85 n_{c}\right)$ plasma slab. Here $n_{c}$ is the critical plasma density for the laser wavelength $\lambda=0.82 \mu \mathrm{m}$. At time $T>0$ a part of the incident laser pulse transmits through the plasma and a significant reflection also occurs. On the contrary in Fig. 4(b) when a similar pulse is incident on an overdense plasma surface of density $n=1.2 n_{c}$ one observes a full reflection from the surface. It is essential to be mentioned here that in both the cases the plasma slabs have been treated with our new hybrid method, i.e., all the plasma particles in this particular simulation were "hybrid particles".

\section{B. Energy Conservation}

Another important point one would like to verify here, is the conservation of the total energy $\left(E_{t o t}\right)$ in the scheme. For this purpose, a test parameter sets with a very trivial laser pulse and plasma systems have been considered. It is important here that we treat the plasma with the hybrid algorithm as well as with kinetic algorithm. The analytical equation for the total energy of the whole system can be written as

$$
E_{t o t}=\sum_{\ell} m_{\ell} c^{2}(\gamma-1)+\frac{1}{8 \pi} \int_{V}\left(E^{2}+B^{2}\right) d V
$$

where, $m_{\ell}$ are the masses of all sort of particles and $\gamma=\sqrt{1+\left(p / m_{\ell} c\right)^{2}}$ is the relativistic $\gamma$ factor. The fields are integrated over all the simulation domain. One can summarize as $E_{t o t}=E_{E M}+E_{\text {part }}$, where, $E_{E M}$ and $E_{\text {part }}$ are total electro-magnetic (EM) and particles' energy. Particles' energy can be estimated as $E_{\text {part }}=n_{e}\left(\gamma_{e}-1\right) m_{e} c^{2}+$ $n_{i}\left(\gamma_{i}-1\right) m_{i} c^{2}+n_{h}\left(\gamma_{h}-1\right) m_{h} c^{2}$, where, $e, i$ and $h$ represent electrons, ions and hybrids respectively. Fig. 5 shows the total energy of the whole laser-plasma system in a closed boundary against time, measured in units of laser periods. The total energy $E_{t o t}$ remains constant nearly up to single precision round off error as it is seen in the inset in Fig. 5 over a significantly large time range. Hence, one can conclude that the total energy in this hybrid scheme is conserved as that of a conventional PIC scheme. 


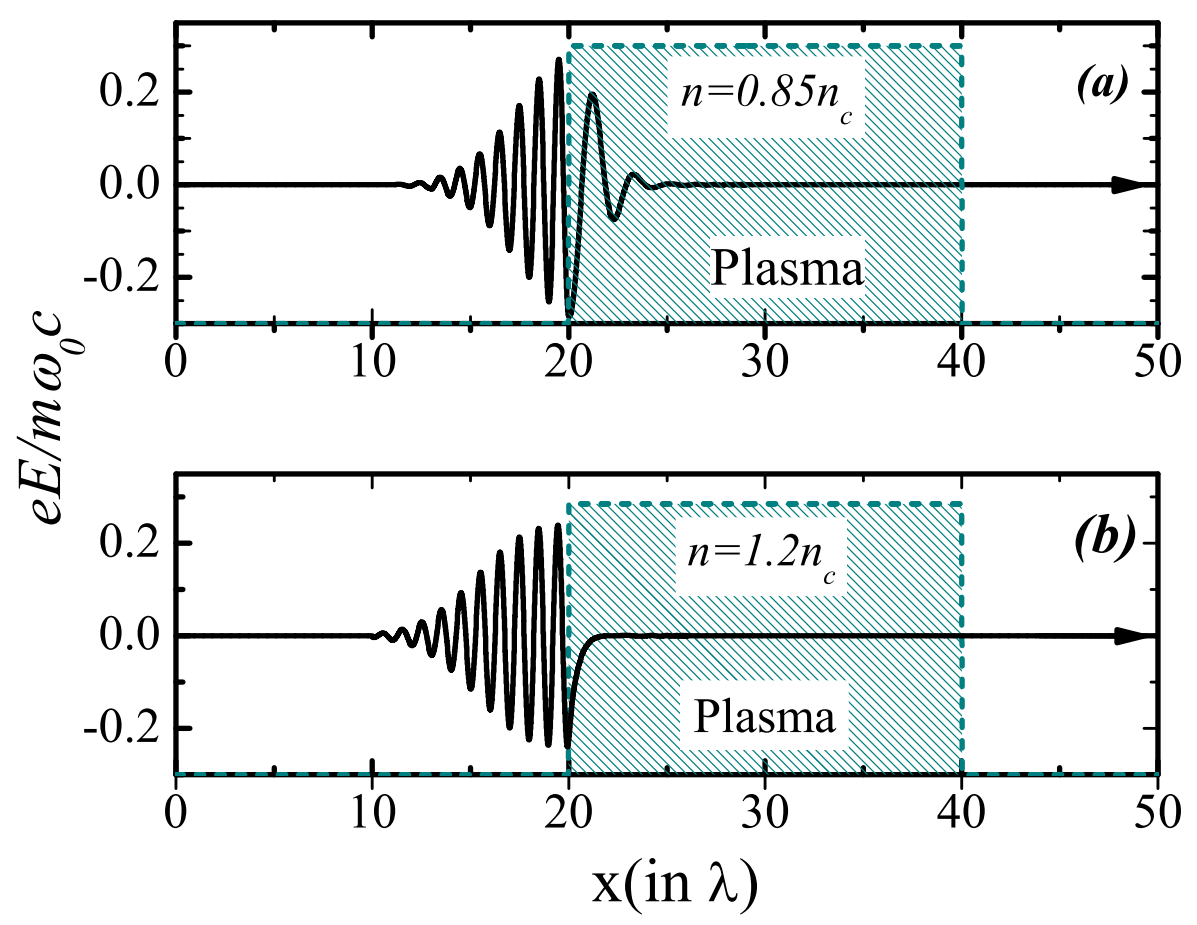

FIG. 4: (color) (a)Refraction of the incident laser pulse through the underdense $\left(n=0.85 n_{c}\right)$ plasma (hybrid model) target. (b) Reflection through overdense $\left(n=1.2 n_{c}\right)$ plasma (hybrid model) target

\section{Acceleration of ions from a target back surface (TNSA)}

Fig. 6 shows the physical model of the generation of ultra-intense energetic protons from laser-solid interactions, first described by Wilks et al.[18]. In the present case, the process has been examined with the new hybrid code. A 10 fs gauss pulse propagating along the positive $X$ direction targeted to a $3.3 \mu \mathrm{m}$ thin slab of plasma considered to be of three components: electrons, ions (protons with $m_{i} / m_{e}=1836$ ) as well as hybrid particles. The density of the target increases to $2 n_{c}$ over a ramp of $\sim 2 \mu \mathrm{m}$. This is to model a good amount of preplasma essentially present in real experiments. In the back surface, there is a thin layer of protons around $\frac{1}{10}$ th of a micron, where the density has been ramped from $2 n_{c}$ to 0 . In reality one can think of a sub-micron sized hydrogen layer pre-formed over the back surface of the target. The dense part of the target was modeled using the hybrid particles with the density $n_{h}=1000 n_{c}$ that would correspond to the solid density. The laser pulse used here has dimensionless amplitude $a_{0}=e E / m \omega_{0} c=2.0$, which corresponds to intensity of $5.5 \times 10^{18} \mathrm{Wcm}^{-2}$.

The ultra short laser pulse interacting with the target generates, in the blow off plasma region, a huge cloud of hot electrons, which propagate through the target and ionize the thin proton layer on the backward surface of the target. These generated protons are then knocked out of the surface by the electrostatic field of the hot electron cloud. Eventually, the ions are accelerated to high energies. In Fig. 7 the energy spectrum of the accelerated ions from the rear surface is graphed with the solid line. The maximum energy reached by these ions are around $\sim 1 \mathrm{MeV}$, which is pretty remarkable energy with the intensity of laser used here.

At this point it is worthy to verify the kinetic nature of the code using a purely kinetic simulation i.e. no hybrid particles. The simulation for the same physical parameters has been performed without the hybrid particles and similar ion energy spectrum has been obtained as that with hybrid particles. This is compared with the hybrid scheme in Fig. 7.

Thus, one can be sure that the hybrid code can be used for computationally efficient studies of very high density plasmas e.g., how to produce mono-energetic ion beams manipulating the thickness of the target as well as the hydrogen layer on the rear surface [20]. To get more insight into the mechanism of acceleration of ions, one can consider looking into the phase space of the hot electron cloud in Fig. 8.

The phase space of the hot electrons (Fig. 8) at an earlier time $T=\omega_{0} t / 2 \pi=100$ (time in laser period) clearly 


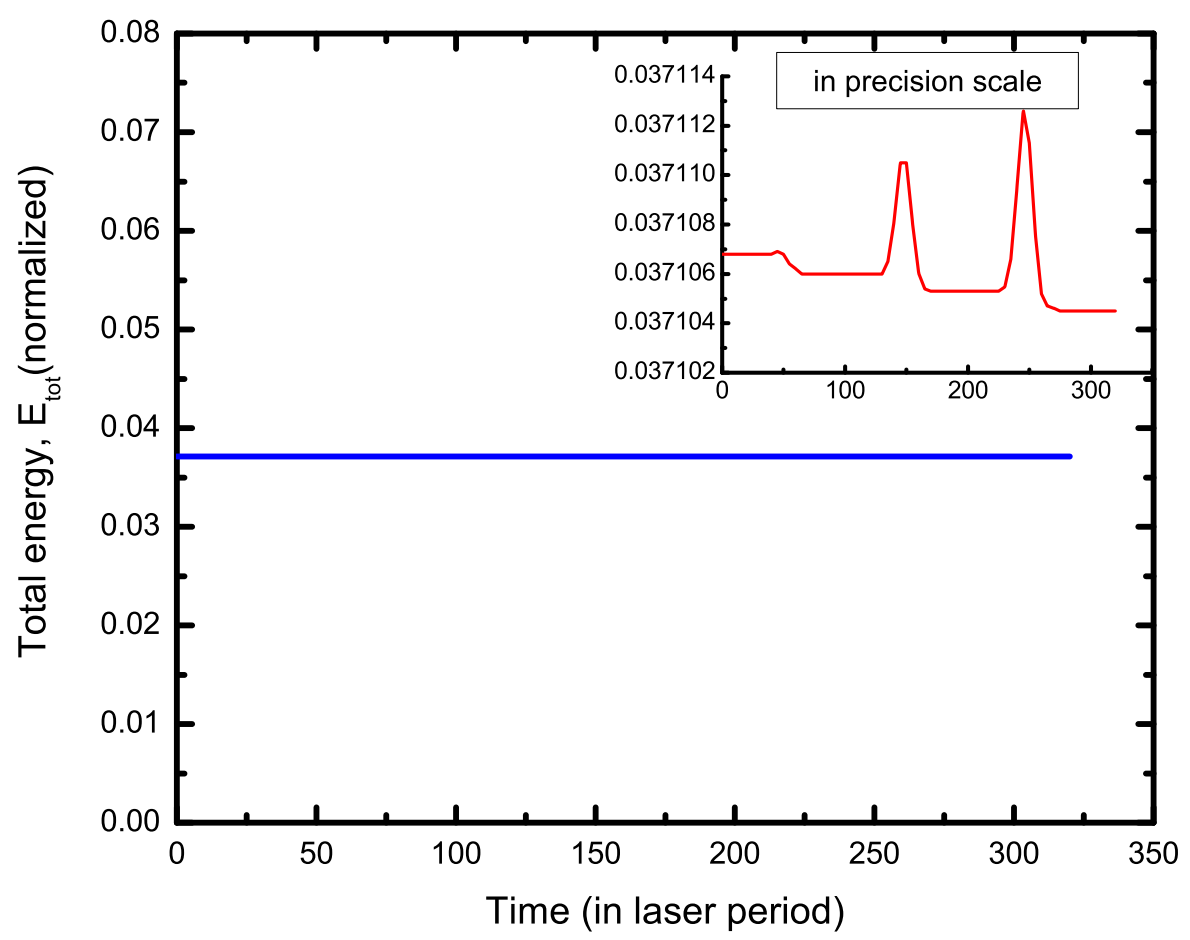

FIG. 5: (color) Total energy conservation in H-VLPL code. The total energy of the whole laser-plasma system has been plotted against laser period. The inset figure is drawn in precision scale of $10^{-6}$ for total energy $E_{t o t}$ with the same data set.

shows that the cloud of hot electrons circulates inside the target and pulls out the ions by significantly heating them in recursion. To get a good amount of acceleration, H-VLPL allows to take as much as 200 particles per cell for such kind of simulations. It is also supposed to get little amount of low energy ions from the front surface, but comparing to that from the rear surface they are colder.

It is proved that most of the acceleration of the ions from the rear surface occur in a short distance. This can also be verified with H-VLPL from the pattern of the accelerating fields of the back surface shown in Fig. 9. In this case this accelerating length is $\sim 4 \mu \mathrm{m}$ and this matches well with experimental and numerical findings observed so far.

At the end one can summarize as, the new hybrid approach of the code H-VLPL benchmarks efficiently well in detail the physics of generation of energetic ions from the target back surface.

\section{Scaling of the skin fields}

To proceed further in benchmarking H-VLPL one can scale the fields at the skin depth $\left(E_{s}\right)$ to the reflected (or incident) fields $\left(E_{i}\right)$. For the present case we choose a range of highly overdense plasmas of densities $n=10,100$ and $1000 n_{c}$ with sharp boundaries. The incident laser is a circularly polarized Gaussian pulse of the dimensionless amplitude $a_{0}=0.2$. Its duration is 10fs. These parameters are chosen to avoid relativistic non-linearities occurring during the interactions.

To benchmark the code we record the laser field at the plasma surface. According to the linear theory, the field $E_{s}$ at the surface of a highly overdense plasma, $n_{e} \gg n_{c}$, relates to the field of the incident laser $E_{i}$ as

$$
E_{s}^{2} / E_{i}^{2}=\frac{4 n_{c}}{n_{e}}
$$

where we have neglected the absorption.

Fig. 10(a) shows the squared ratio of the field, $E_{s}{ }^{2} / E_{i}{ }^{2}$ as a function of the normalized plasma density $n=n_{e} / n_{c}$. The numerical results obtained from the H-VLPL code matches well with the analytical result (27). 


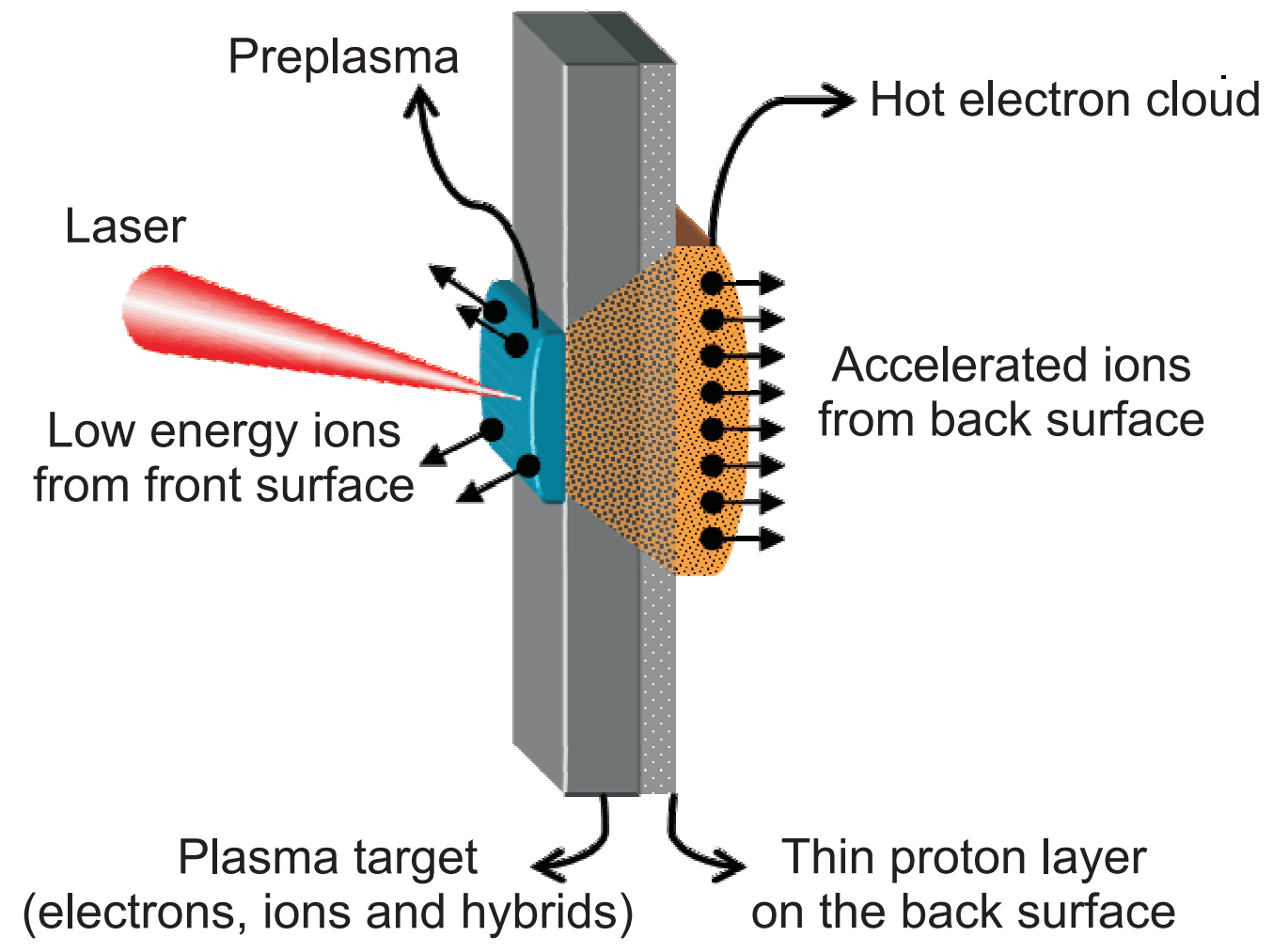

$X(\lambda)$

FIG. 6: (color) Physical model of ion acceleration mechanism for a short and ultra-intense pulse interacting with thin target.

On the other hand, we know that the laser fields must decay exponentially in the overdense plasma layer. Analytically, the electromagnetic field intensity decays as $\ln I=-2 x / \delta_{s}$, where $\delta_{s}=c / \omega_{p}$ is the skin depth. We have measured the field decay in the H-VLPL simulations and plotted them in Fig. 10(b). One gets excellent agreement with the analytical expressions for the skin length even for the highest densities.

For the density $n_{e}=1000 n_{c}$, one finds the skin length $\delta_{s} / \lambda=0.005$. It is worth mentioning that this simulation has been done with the grid cell size $h_{x} / \lambda=0.05$, i.e, the grid step was much larger than the skin length, $h_{x}=10 \delta_{s}$. Yet, the field decay in plasma is accurately described.

\section{E. Collisional Absorption}

We have introduced the effects of collisional absorption into the implicit hybrid scheme of H-VLPL. This makes the code versatile enough to handle extremely high density warm plasmas where electron-ion binary collisions cannot be neglected. However, to test the accuracy of the collisional scheme we have chosen laser pulse absorption as it propagates in underdense plasma. This is because there is a known analytic solution for the laser dynamics to compare with.

Fig. 11 shows the change in laser amplitude as it propagates through a collisional underdense plasma of density $n=0.04 n_{c}$. The laser pulse is chosen to be weakly relativistic, $a_{0}=0.2$, and relatively long Gaussian pulse of $50 \mathrm{fs}$ duration. The longer duration is selected to avoid dispersion effects. The collisional frequency $\nu_{e i}$ (described as $\eta$ in Section II) is 0.5 . We calculated the logarithmic field amplitudes at various propagation lengths, $x$ from H-VLPL and compare the results with the analytical solution

$$
\ln \left(E / E_{0}\right)=\operatorname{Re}[-i \omega t+i k x-\Delta x]
$$




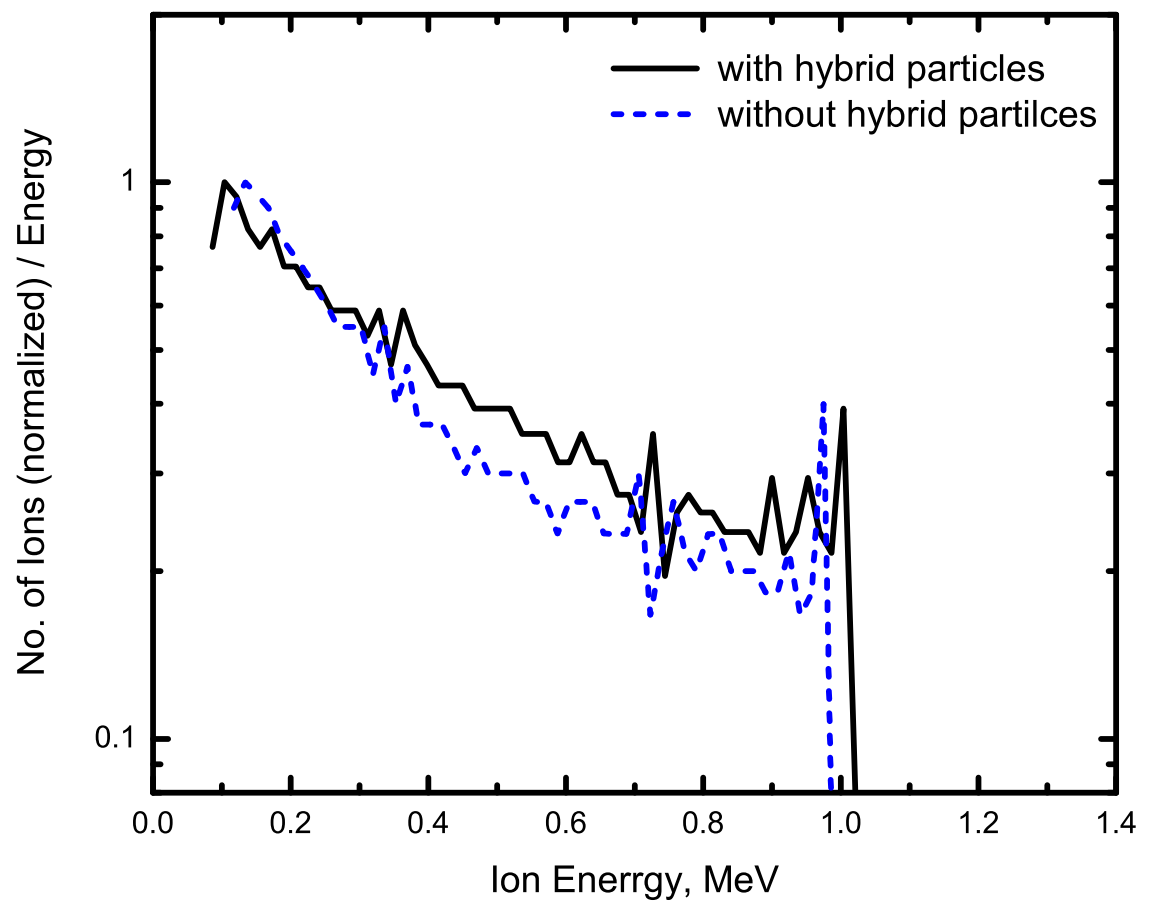

FIG. 7: (color) Energy spectrum of the accelerated ions from rear surface of the target (solid line)as shown in Fig. 6 at a later time $T=\omega_{0} t / 2 \pi=150$. Ions' kinetic energy is in MeV scale. The maximum kinetic energy of the ions is $\sim 1 \mathrm{MeV}$. The broken line reproduces the similar ion spectrum in fully kinetic environment where no hybrid particles are present, at the same reference time.

where

$$
\Delta \approx \frac{1}{2} \frac{\omega_{p}^{2}\left(\nu_{e i} / \omega\right)}{\left(\omega^{2}-\omega_{p}^{2}\right)^{1 / 2}}
$$

is the collisional absorption rate. The simulation gives an exponentially decreasing laser field $E$ inside the bulk plasma as it propagates along. These results are shown in Fig. 11(a). Also, the decreasing peak intensity over laser propagation time (in laser period) in Fig. 11(b) confirms the effect of collisional absorption in agreement with the analytic solution.

\section{CONCLUSIONS AND FUTURE PLANS}

In conclusion, we have presented the implicit hybrid algorithm that allows to simulate laser-plasma interactions at arbitrary plasma densities. It avoids the limitation on the time step present in explicit PIC codes. The numerical scheme is analyzed, its dispersion relation is derived, and a numerically efficient matrix algorithm for solving the implicit system of equations is presented.

The scheme is tested on a series of physically important examples. It is shown that the spatial field structure in the highly overdense plasma is well described by the code H-VLPL even when the grid step size is much larger than the plasma skin length.

The presented scheme is one dimensional. The next step will be to generalize this hybrid algorithm to the full three dimensional geometry. 


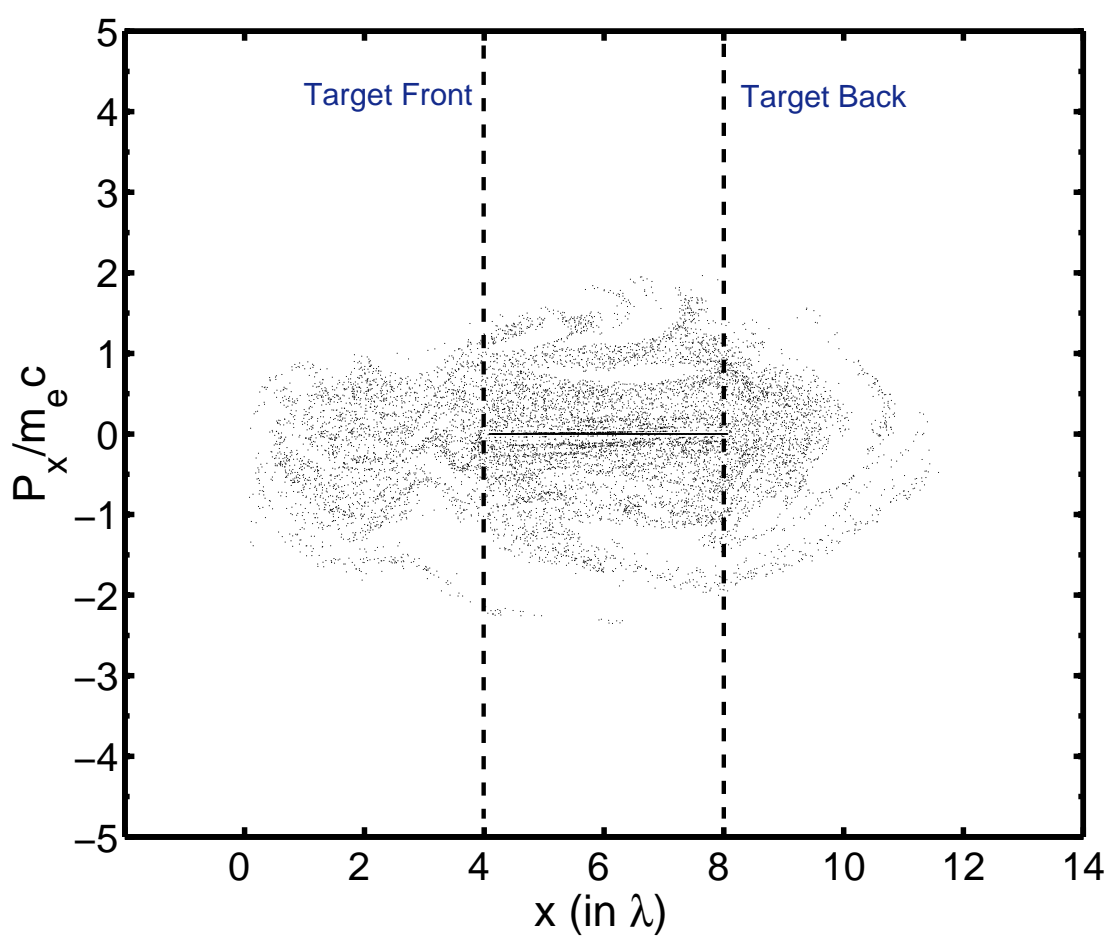

FIG. 8: (color) Phasespace diagram of the hot electron cloud at an earlier time $T=\omega_{0} t / 2 \pi=100$. Electron momentums are normalized to $m_{e} c$ and $x$ axis in units of $\lambda$. The broken lines represents the plasma target front and back surfaces as that of the schematic of Fig. 6.

This work was supported by the DFG through project TR-18.

[1] C. K. Birdsal, A. B. Langdon, Plasma Physics Via Computer Simulation, Adam Higer, (1991).

[2] R. W. Hockney, J. W. Eastwood, Computer Simulation Using Particles, McGraw-Hill, London, (1981).

[3] Pukhov. A, J. Plasma Phys. 61 (1999) 425.

[4] R. A. Fonseca, L. O. Silva, F. S. Tsung, V. K. Decyk, W. Lu, C. Ren, W. B. Mori, S. Deng, S. Lee, T. Katsouleas, J. C. Adam, OSIRIS: A Three-Dimensional, Fully Relativistic Particle in Cell Code for Modeling Plasma Based Accelerators, in: Lecture Notes in Computer Science 2331 (2002) 342.

[5] C. Nieter, J. R. Cary, J. Comput. Phys. 196(2004) 448.

[6] J. P. Verboncoeur, A. B. Langdon, N. T. Gladd, Comput. Phys. Commun. 196(2004) 448.

[7] L. Robson, P. T. Simpson, R. J. Clarke, K. W. D. Ledingham, F. Lindau, O. Lundh, T. McCanny, P. Mora, D. Neely, C.-G. Wahlström, M. Zepf and P. McKenna, Nature Physics 3 (2007) 58; M. Roth, et al. Phys. Rev. Special TopicsAccelerators and Beams 5 (2002) 061301; M. Kaluza et al. Phys. Rev. Lett. 93 (2004) 045003.

[8] M. Tabak, J. H. Hammer, M. E. Glinsky, W. L. Kruer, S. C. Wilks, J. Woodworth, E. M. Campbell, M. D. Perry, and R. J. Mason, Phys. Plasmas 1, (1994) 1626.

[9] R. J. Mason, Phys. Fluids 23 (1980) 2204.

[10] P. W. Rambo and J. Denavit, J. Comp. Phys. 92, (1991) 185.

[11] P. W. Rambo and J. Denavit, Phys. Plasmas 1, (1994) 4050.

[12] J. R. Davies, A. R. Bell, M. G. Haines, and S. Guerin, Phys. Rev. E 56 (1997) 7193.

[13] L. Gremillet, G. Bonnaud, F. Amiranoff, Phys. Plasmas 9 (2002) 941.

[14] D. R. Welch, D. V. Rose, R. E. Clark, T. C. Genoni, T. P. Hughes Comput. Phys. Commun., 164(2004) 183.

[15] F. Zheng, Z. Chen, IEEE Trans. Microwave Theory Tech. 49 (2001) 1006.

[16] N. A. Krall and A. W. Trivelpiece, Principles of Plasma Physics, McGraw-Hill, N.Y. (1973).

[17] G. H. Golub, C. Van L., Matrix Computations, Johns Hopkins University Press, 3rd edition (1996)

[18] S. C. Wilks, A. B. Langdon, T. E. Cowan, M. Roth, M. Singh, S. Hatchett et al. Phys. Plas, 8(2001) 542.

[19] A. Pukhov, Phys. Rev. Lett. 86 (2001) 3562.

[20] N. Kumar, A. Pukhov, arXiv:0710.3019v2 [physics.plasm-ph], (2007). 


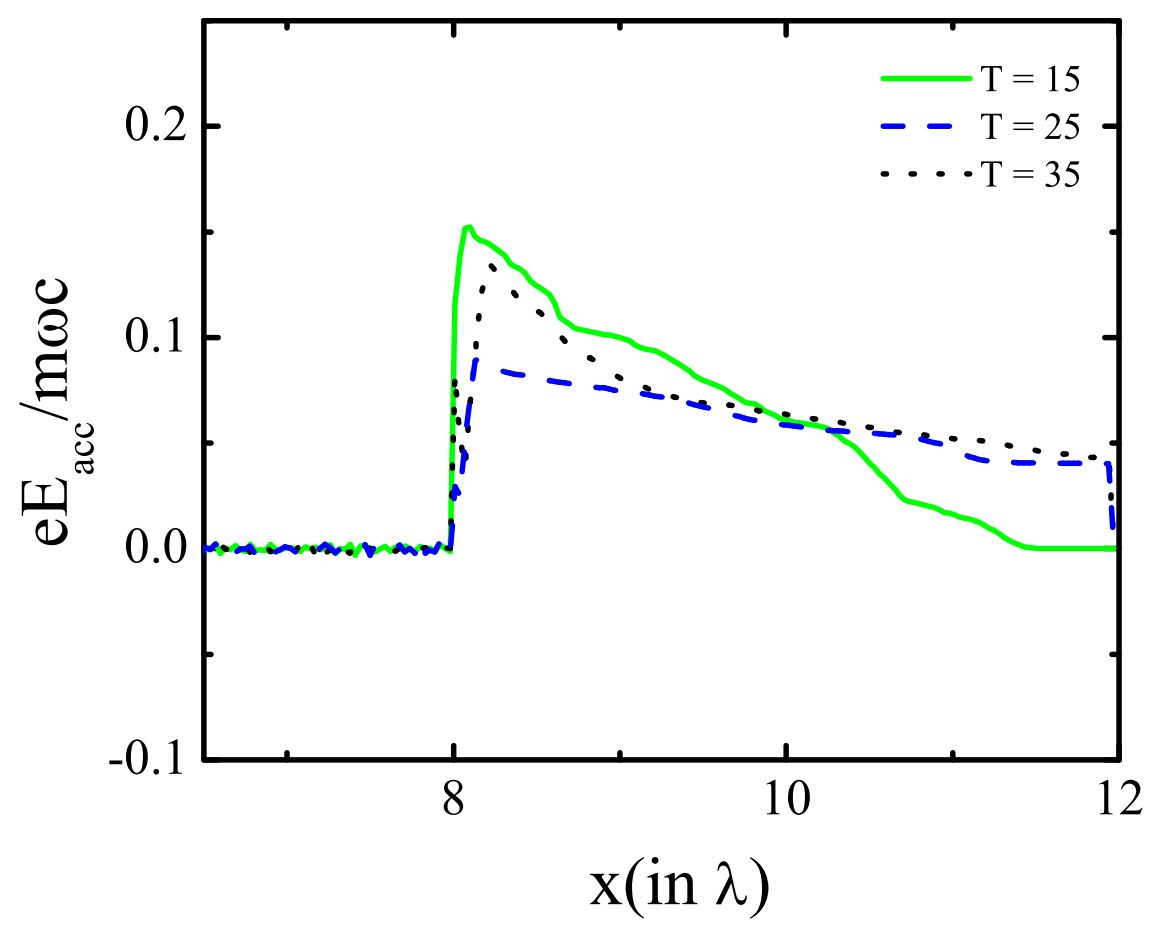

FIG. 9: (color) The accelerating electric fields, at three different earlier times $T=\omega_{0} t / 2 \pi=15,25$ and 35 .
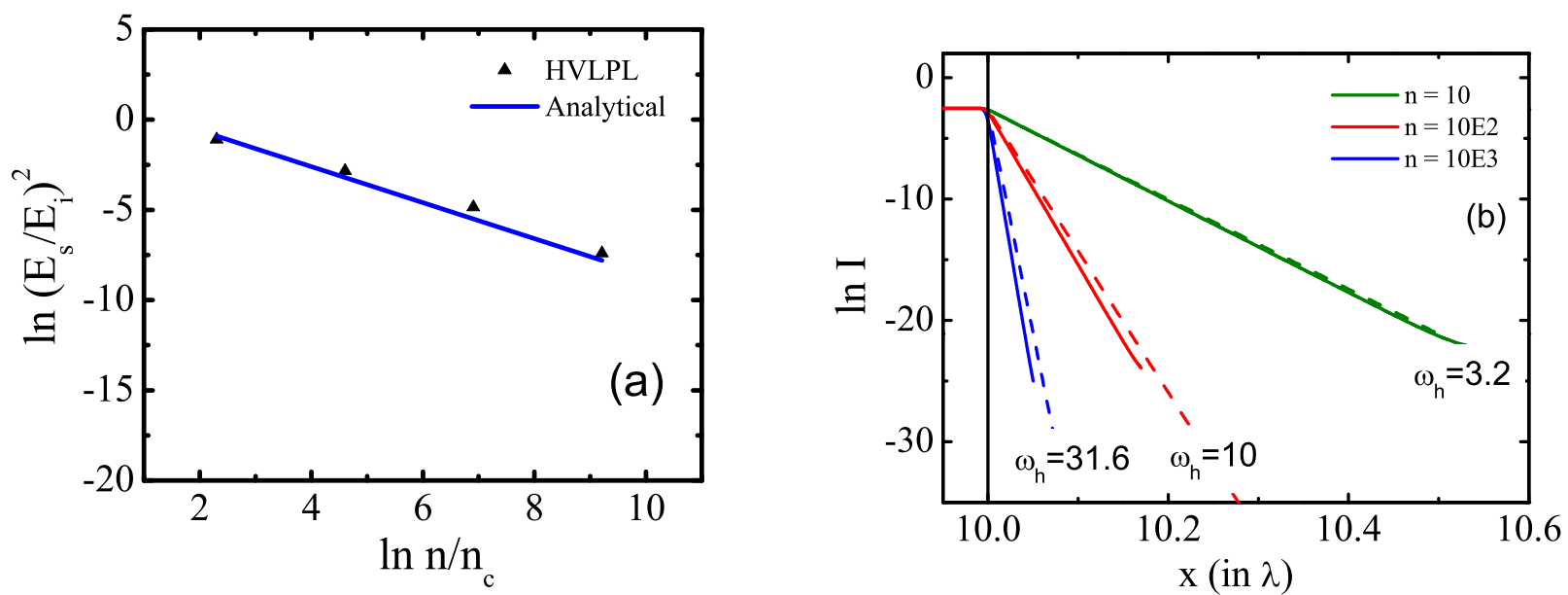

FIG. 10: (color) (a) Logarithm of skin fields plotted for four different hybrid densities. The fields are normalized as $e E / m \omega_{0} c$ and density to $n_{c}$. The solid line shows the analytical results for the same. (b) Logarithm of skin field intensities for three different hybrid densities. The analytically calculated values of $\omega_{p}$ are also provided for each densities for comparison. The dashed lines show the analytical results for the corresponding densities. The dispersion relation for $\omega_{h}=31.6$ has been discussed earlier in Fig. 3. 

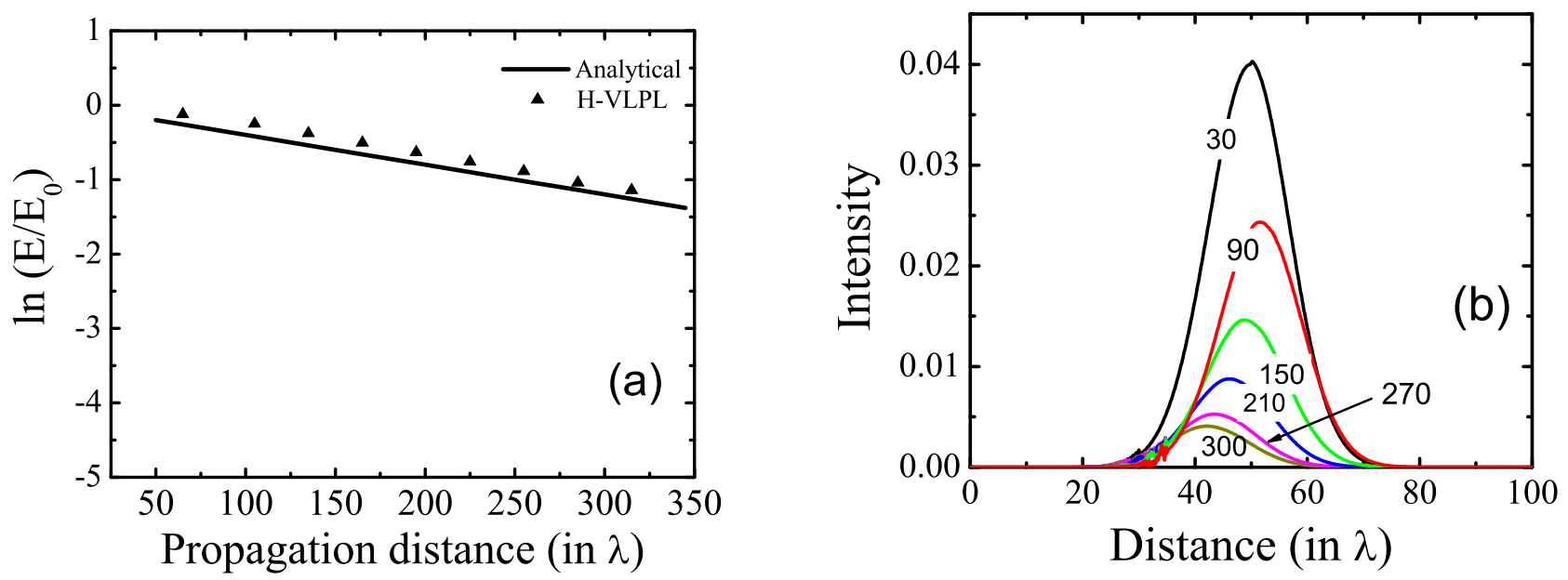

FIG. 11: (color) Collisional absorption: (a) Logarithm damping of laser amplitude $E$ along the propagation direction and comparison with analytical results (b) Damping of incident laser intensity over time; all the times are in scale of laser periods. 